\title{
Prostaglandin Synthetase Inhibition in Group B Streptococcal Shock: Hematologic and Hemodynamic Effects
}

\author{
KEITH J. PEEVY, PETER PANUS, GESINA L. LONGENECKER, STEPHEN A. CHARTRAND, \\ HOLLIS J. WISEMAN, ROBERT C. BOERTH, AND RICHARDSON D. OLSON
}

Departments of Pediatrics and Pharmacology, University of South Alabama, College of Medicine, Mobile, Alabama 36617

\begin{abstract}
A rabbit model of group B Streptococcal (GBS) shock was used to study the effects of prostaglandin synthetase inhibition on the hemodynamic and hematologic response to GBS shock. The infusion of heat-killed GBS in groups I and II produced significant decreases in mean arterial pressure, neutrophil counts, and platelet counts ( $p$ $<0.05$ ), and significant rises in concentrations of thromboxane $B_{2}$ and 6-KetoPGFla, the stable metabolites of thromboxane $A_{2}$ and prostacyclin $(p<0.05)$. Administration of indomethacin $(4 \mathrm{mg} / \mathrm{kg}$ ) after GBS infusion (group II) was associated with a significant rise in mean arterial pressure and a significant decline in thromboxane $B_{2}$ and 6-Keto-PGFla concentrations $(p<0.05)$ but had no effect on GBS-induced hematologic alterations. Indomethacin administration before GBS infusion (group III) prevented alterations in mean arterial pressure and was associated with a decrease in thromboxane $B_{2}$ and 6-Keto-PGFla concentrations. Indomethacin in group III did not prevent neutropenia and thrombocytopenia and may have exacerbated neutropenia. Alteration of experimental GBS shock with prostaglandin synthetase inhibition produces disparate hemodynamic and hematologic response. (Pediatr Res 20:864-866, 1986)
\end{abstract}

\section{Abbreviations}

GBS, group B Streptococcus

PG, prostaglandin

IND, indomethacin

$\mathrm{TB}_{2}$, thromboxane $\mathrm{B}_{2}$

6-KPGFla, 6-Keto-PGFla

MAP, mean arterial pressure

CFU, colony forming units

Neonatal GBS sepsis produces severe hemodynamic and hematologic manifestations (1). The clinical findings of shock, acidosis, neutropenia, and marrow granulocyte depletion are thought to be correlates of high mortality in GBS sepsis (2). Although arachidonic acid metabolites have been strongly implicated as modulators of the septic shock process (3), only Rojas et al. (4) have directed specific attention to possible interactions between PG endoperoxide products and the hematologic alterations of GBS sepsis. In their sheep lung lymph model, an extract

Received November 7, 1985; accepted April 25, 1986.

Reprint Requests Dr. Keith J. Peevy, Department of Pediatrics, University of South Alabama, 2451 Fillingim Street, Mobile, AL 36617.

This work was funded by a grant from the Alabama Chapter American Heart Association and by NIH Grant HL28711. of GBS produced granulocytopenia which was not altered by the prior administration of IND. These data suggest a lack of association between PG endoperoxide products and the development of neutropenia. However, this model was not designed to produce septic shock and therefore it could not be used to evaluate the possible influence of PG synthetase inhibition on neutropenia through modulation of septic shock. In addition, no studies have evaluated the possibility that PG synthetase inhibitors might exacerbate GBS-associated hematologic changes.

The purpose of this study was to define the role of PG endoperoxide products in modulating the hemodynamic and hematologic alterations of experimental GBS shock, and to further define how PG synthetase inhibition might alter the manifestations of the shock process.

\section{MATERIALS AND METHODS}

Preparation of $G B S$. A strain of GBS isolated from the blood of an infant dying with shock was used in all studies. Bacterial isolates were frozen at $-20^{\circ} \mathrm{C}$ between isolation and use in studies. Before each series of experiments the organism was passed in a mouse, and isolated colonies from a heart blood subculture were grown overnight on blood agar. Bacteria were suspended in sterile, nonpyrogenic saline to a quantified density of $10^{11} \mathrm{CFU}$ per $\mathrm{ml}$ and then heat-killed at $100^{\circ} \mathrm{C}$ for $15 \mathrm{~min}$. CFU were determined by quantitative subcultures, and complete heat-killing was documented by subculturing after heat killing.

Animal preparation. Following intravenous pentobarbitol anesthesia $(50 \mathrm{mg} / \mathrm{kg})$, adult New Zealand White rabbits underwent tracheostomy and were placed on a small animal ventilator. Femoral arterial and venous catheters were inserted for measurement of the following: MAP, heart rate, arterial blood gases, total leukocyte counts annd differentials, platelet counts, and concentrations of $\mathrm{TB}_{2}$ and $6 \mathrm{KPGFla}$, the stable metabolites of thromboxane $\mathrm{A}_{2}$ and prostacyclin, respectively. Animals were hyperventilated to an alkalotic $\mathrm{pH}$ to minimize the anticipated effects of GBS-induced acidosis on MAP.

Experimental sequence. Four groups of animals were studied. In groups I $(n=8)$ and II $(n=9)$ basal values for MAP, TB $\mathrm{TB}_{2}, 6$ KPGFla, neutrophil count, and platelet count were obtained, followed by infusion of heat-killed GBS $\left(10^{12}\right.$ organisms $\left./ \mathrm{kg}\right)$ over $60 \mathrm{~min}$. After MAP fell by $50 \%$, repeat samples for $\mathrm{TB}_{2}, 6-$ KPGFla, neutrophil count, and platelet count were obtained. This was followed by the administration of IND $(4 \mathrm{mg} / \mathrm{kg}$ ) (group II) or drug vehicle (group I). Additional samples for determination of $\mathrm{TB}_{2}$ and 6-KPGFla levels, and neutrophil and platelet counts were obtained $60 \mathrm{~min}$ after IND or vehicle infusion.

In group III $(n=7) \mathrm{MAP}, \mathrm{TB}_{2}$, and 6-KPGFla concentrations, and hematologic values were obtained in the basal state, after IND pretreatment $(4 \mathrm{mg} / \mathrm{kg})$, and 60 min after initiation of GBS 
infusion. Control animals (group IV, $n=6$ ) were sampled for the same hemodynamic, hematologic, and PG variables in the basal state, $60 \mathrm{~min}$ after the initiation of a sterile, nonpyrogenic saline infusion, and $60 \mathrm{~min}$ after drug vehicle infusion.

Laboratory determinations. Total white blood cell counts and platelet counts were determined on a model $\mathrm{S}^{+}$Coulter Counter. Peripheral blood absolute neutrophil counts were performed by a registered medical technologist. $\mathrm{TB}_{2}$ and 6-KPGFla concentrations were assayed using standardized radioimmunoassay kits from New England Nuclear.

Statistical evaluation. Statistical comparisons of data were performed using one-way analysis of variance and randomized block design analysis of variance for unpaired and paired comparisons respectively, Duncan's test, and $\chi^{2}$ analysis. A $p$ value of $<0.05$ was considered significant.

\section{RESULTS}

Table 1 lists the results for MAP, $\mathrm{TB}_{2}, 6-\mathrm{KPGFla}$, neutrophil counts, and platelet counts for the four study groups. Mean arterial pressure fell significantly after GBS infusion in groups I and II. Administration of drug vehicle in group I did not alter the MAP, but in group II animals IND administration produced a significant rise of MAP to near the basal state. Animals infused with GBS after pretreatment with IND (group III) and those infused only with saline (group IV) showed no significant alterations of MAP. In groups I and II, infusion of GBS produced increases in $\mathrm{TB}_{2}$ and 6-KPGFla concentrations which were significantly elevated above the basal value. In group $\mathrm{I}, \mathrm{TB}_{2}$ and 6KPGFla concentrations remained elevated after drug vehicle infusion, although only the 6-KPGFla value was significantly different from the basal state. In group II IND treatment resulted in a significant decline in $\mathrm{TB}_{2}$ and 6-KPGFla concentrations. Levels of both PG endoperoxide products fell from basal values in group III after IND infusion, even in the presence of GBS administration. Control animals showed no changes in concentrations of either PG endoperoxide product.

In groups I, II, and III both neutrophil and platelet counts fell dramatically after the administration of GBS. Neither pre- (group III) nor post-GBS (group II) administration of IND appeared to prevent the significant fall in these hematologic values. IND pretreatment in group III was associated with a significantly lower neutrophil count after GBS infusion than when only GBS and drug vehicle were given (group I) $(p<0.05)$. In addition, animals in groups I and II had significantly less frequent total neutrophil depletion than in group III (data not shown). Group IV animals demonstrated a statistically significant rise in neutrophil count and a modest decline in platelet count. In none of the groups was a significant change in immature neutrophil count or immature/mature neutrophil ratio found.

Table 2 provides arterial $\mathrm{pH}$, blood gases, and calculated bicarbonate concentrations on the four groups. A significant

Table 2. Arterial blood gas, $p H$, and calculated bicarbonate values at baseline and after each intervention (mean $\pm S E$ )

\begin{tabular}{lcccc}
\hline \multicolumn{1}{c}{ Group } & \multicolumn{1}{c}{$\mathrm{pH}$} & $\begin{array}{c}\mathrm{pO}_{2} \\
\text { (torr) }\end{array}$ & $\begin{array}{c}\text { pco } 2 \\
\text { (torr) }\end{array}$ & $\begin{array}{c}\text { Calculated } \\
\mathrm{HCO}_{3} \\
\text { (mEq/ } \\
\text { liter) }\end{array}$ \\
\hline I $(n=8)$ & & & & \\
Baseline & $7.67 \pm 0.03$ & $78 \pm 4$ & $22 \pm 2$ & $24 \pm 2$ \\
GBS & $7.44 \pm 0.05^{*}$ & $58 \pm 6^{*}$ & $25 \pm 3$ & $16 \pm 1^{*}$ \\
Drug vehicle & $7.32 \pm 0.04 \dagger$ & $70 \pm 7$ & $22 \pm 3$ & $11 \pm 2 \dagger$ \\
& & & & \\
II $(n=9)$ & & & & \\
Baseline & $7.64 \pm 0.03$ & $85 \pm 4$ & $23 \pm 1$ & $23 \pm 1$ \\
GBS & $7.35 \pm 0.03^{*}$ & $48 \pm 4 \dagger$ & $33 \pm 1^{*}$ & $17 \pm 1^{*}$ \\
IND & $7.33 \pm 0.06^{*}$ & $87 \pm 7$ & $26 \pm 1 \dagger$ & $14 \pm 2 \dagger$ \\
III $(n=7)$ & & & & \\
Baseline & $7.66 \pm 0.04$ & $73 \pm 6$ & $25 \pm 2$ & $26 \pm 1$ \\
IND & $7.63 \pm 0.04$ & $75 \pm 5$ & $25 \pm 2$ & $25 \pm 1$ \\
IND + GBS & $7.49 \pm 0.01 \dagger$ & $79 \pm 5$ & $28 \pm 2$ & $20 \pm 1 \dagger$ \\
& & & & \\
IV $(n=6)$ & & & & \\
Baseline & $7.61 \pm 0.02$ & $75 \pm 4$ & $27 \pm 2$ & $26 \pm 1$ \\
Saline & $7.59 \pm 0.03$ & $72 \pm 11$ & $28 \pm 3$ & $25 \pm 1$ \\
Drug vehicle & $7.58 \pm 0.02$ & $69 \pm 4$ & $27 \pm 2$ & $24 \pm 2^{*}$ \\
\hline
\end{tabular}

* Denotes significant difference from base within group.

$\dagger$ Denotes significant difference from both other values within group.

Table 1. Baseline and postintervention values for $M A P, T B_{2}, 6 K P G F 1 a$, neutrophils, and platelets (mean $\left.\pm S E\right)$

\begin{tabular}{|c|c|c|c|c|c|}
\hline Group & $\begin{array}{c}\text { MAP } \\
(\mathrm{mm} \mathrm{Hg})\end{array}$ & $\mathrm{TB}_{2}(\mathrm{pg} / \mathrm{ml})$ & $\begin{array}{l}\text { 6KPGFla } \\
(\mathrm{pg} / \mathrm{ml})\end{array}$ & $\begin{array}{l}\text { Neutrophils } \\
\left(\times 10^{3} / \mathrm{mm}^{3}\right)\end{array}$ & $\begin{array}{c}\text { Platelets } \\
\left(\times 10^{3} /\right. \\
\left.\mathrm{mm}^{3}\right)\end{array}$ \\
\hline \multicolumn{6}{|l|}{$\mathrm{I}(n=8)$} \\
\hline Baseline & $88 \pm 3$ & $48.1 \pm 8.0$ & $18.9 \pm 6.2$ & $3.5 \pm 0.5$ & $376 \pm 55$ \\
\hline GBS & $49 \pm 7^{*, \dagger}$ & $80.8 \pm 8.5^{* \cdot \dagger}$ & $91.1 \pm 27.3^{*, \dagger}$ & $0.8 \pm 0.2^{*, \dagger}$ & $145 \pm 51^{*}$ \\
\hline Drug vehicle & $39 \pm 7^{*, \dagger}$ & $75.3 \pm 11.8 \dagger$ & $113.9 \pm 15.7^{*, \dagger}$ & $0.5 \pm 0.2^{*, \dagger}$ & $119 \pm 46^{*}$ \\
\hline \multicolumn{6}{|l|}{ II $(n=9)$} \\
\hline Baseline & $98 \pm 4$ & $35.6 \pm 4.8$ & $15.3 \pm 3.0$ & $2.2 \pm 0.6$ & $365 \pm 29$ \\
\hline GBS & $47 \pm 5 \ddagger$ & $74.6 \pm 15.3 \ddagger$ & $92.3 \pm 2.7 \ddagger$ & $0.2 \pm 0^{*}$ & $117 \pm 38^{*}$ \\
\hline IND & $87 \pm 6$ & $27.2 \pm 4.8$ & $30.9 \pm 10.2$ & $0.2 \pm 0.1^{*}$ & $111 \pm 37^{*}$ \\
\hline \multicolumn{6}{|l|}{ III $(n=7)$} \\
\hline Baseline & $89 \pm 5$ & $40.1 \pm 4.3$ & $28.7 \pm 5.9$ & $2.5 \pm 0.5$ & $338 \pm 68$ \\
\hline IND & $86 \pm 6$ & $13.7 \pm 6.6^{*}$ & $21.2 \pm 6.9$ & $1.9 \pm 0.5$ & $389 \pm 53$ \\
\hline $\mathrm{IND}+\mathrm{GBS}$ & $84 \pm 5 \dagger$ & $8.7 \pm 1.5^{*, \dagger}$ & $13.8 \pm 4.2 \dagger$ & $0 . \pm 0 \dagger, \ddagger$ & $69 \pm 21 \ddagger$ \\
\hline \multicolumn{6}{|l|}{$\operatorname{IV}(n=6)$} \\
\hline Baseline & $92 \pm 5$ & $33.2 \pm 3.5$ & $10.5 \pm 2.5$ & $2.6 \pm 0.5$ & $423 \pm 84$ \\
\hline Saline & $90 \pm 4$ & $25.0 \pm 3.3$ & $17.7 \pm 3.0$ & $4.8 \pm 0.7^{*}$ & $394 \pm 70$ \\
\hline Drug vehicle & $85 \pm 4$ & $30.3 \pm 6.3$ & $14.0 \pm 6.1$ & $5.1 \pm 1.0^{*}$ & $364 \pm 82 *$ \\
\hline
\end{tabular}

* Denotes significant difference from base within group $(p<0.05)$.

† Denotes significant difference between post-GBS values in groups I and III $(p<0.05)$.

$\ddagger$ Denotes significant difference from both other values within group $(p<0.05)$. 
decline in pH occurred in groups I, II, and III after administration of GBS although the magnitude of decline was less in the INDpretreated animals (group III). A significant fall in $\mathrm{PaO}_{2}$ occurred in groups $\mathrm{I}$ and $\mathrm{II}$ and a significant rise in $\mathrm{PaCO}_{2}$ occurred in group II. No statistically significant changes in $\mathrm{PaO}_{2}$ or $\mathrm{PaCO}_{2}$ occurred in IND-pretreated animals or saline-infused controls. All groups showed a significant decline in calculated bicarbonate values over time. However, the magnitude of the decline was greatest in groups I and II, a finding consistent with the alterations in $\mathrm{pH}$ in those groups.

\section{DISCUSSION}

Several investigators have shown an association between PG endoperoxide products or PG synthetase inhibition and the hemodynamic manifestations of GBS shock (5-7). Our present data demonstrate that in experimental GBS shock systemic hypotension is associated with changes in levels of PG metabolites, and that these changes are preventable or significantly altered by IND treatment. In group I, drug vehicle values for $\mathrm{TB}_{2}$ are not significantly different from baseline within the group, but were significantly different from the values for the other three groups. This late fall in $\mathrm{TB}_{2}$ in group I may reflect poststimulation exhaustion of platelet $\mathrm{TB}_{2}$ production. The significant fall in $\mathrm{TB}_{2}$ in group II after indomethacin suggests that a major reduction in $\mathrm{TB}_{2}$ production is still achievable through PG synthetase inhibition.

The relationships of peripheral neutrophil and platelet depletion to the development of GBS-shock and PG production have not been well studied. Our data demonstrate three important points in this regard: 1) the lack of correlation between $\mathrm{TB}_{2}$ and 6-KPGFla concentrations and neutrophil count in group II and the development of neutropenia in group III support the idea that neutropenia occurs independently of PG production; 2) neutropenia in group III demonstrates that shock is not a necessary antecedent of GBS-induced neutropenia; and 3) a stable MAP in group III demonstrates that shock is not a sequelae of GBS-induced neutropenia.

In group III, the lower mean neutrophil count and the greater frequency of total peripheral neutrophil depletion could be chance occurrences of no clinical or scientific significance. How- ever, they suggest that inhibition of PG synthetase prior to GBS administration may alter peripheral neutrophil removal. In vitro studies showing IND enhancement of arachidonate-induced neutrophil aggregation (8) and IND inhibition of GBS-induced neutrophil aggregation (9) lend conflicting information, but support the idea that PG metabolism may affect the aggregation process.

The significant rise in neutrophil count in group IV was thought to be due to demargination induced by the stress of anesthesia and instrumentation.

In summary, PG synthetase inhibition with IND reduced GBSinduced production of PG endoperoxide products and altered the hemodynamic response to experimental GBS shock. GBSinduced neutropenia and thrombocytopenia was not prevented by IND, although pretreatment with IND may have enhanced peripheral neutrophil depletion. Further in vitro and in vivo studies are needed to elucidate the role of PG synthetase inhibitors in modulating the hematologic response to GBS shock.

\section{REFERENCES}

1. Baker C 1977 From the National Institutes of Health: Summary of the workshop in perinatal infections due to group B Streptococcus. J Infect Dis 136:137-152

2. Christensen R, Bradley P, Rothstein G 1981 The leukocyte left shift in clinical and experimental neonatal sepsis. J Pediatr 98:101-105

3. Hess M, Hastillo A, Greenfield L 1981 Spectrum of cardiovascular function during gram-negative sepsis. Prog Cardiovasc Dis 23:279-298

4. Rojas J, Larsson L, Ogletree M, Brigham K, Stahlman M 1983 Effects of cyclooxygenase inhibition on the response to group B streptococcal toxin in sheep. Pediatr Res 17:107-110

5. Runkle B, Goldberg R, Streitfeld M, Clark M, Buron E, Setzer E, Bancalari E 1984 Cardiovascular changes in group B streptococcal sepsis in the piglet: response to indomethacin and relationship to prostacyclin and thromboxane $A_{2}$. Pediatr Res 18:874-878

6. Lunyong V, Smith S 1983 Thromboxane and pulmonary hypertension following group $B$ beta strep infusion in newborn piglets: inhibiting effect of indomethacin. Pediatr Res 17:382A

7. Peevy K. Chartrand S, Wiseman H, Boerth R, Olson R 1985 Myocardial dysfunction in group B streptococcal shock. Pediatr Res 19:511-513

8. Panus $P$, Longenecker G 1984 Sodium arachidonate induced in vitro polymorphonuclear leukocyte aggregation. Res Commun Chem Pathol Pharmacol 43:393-405

9. Panus P, Longenecker G, Chartrand S, Boerth R, Peevy K 1985 Comparison of the effects of various agents on in vitro neutrophil aggregation initiated by opsonized heat inactivated group B streptococcus or arachidonic acid. J Clin Pharmacol 25:635A 